\title{
Discovering Dominant Users' Opinions in Reddit
}

\author{
Teresa ALSINET ${ }^{1}$, Josep ARGELICH, Ramón BÉJAR and Santi MARTÍNEZ \\ INSPIRES Research Center - University of Lleida \\ Jaume II, 69 - 25001 Lleida, SPAIN
}

\begin{abstract}
Reddit is a social news aggregation and discussion website. Users submit content to the site such as links to news, which are then voted up or down by other members who in turn, can comment on others' posts to continue the conversation. In this work, we are interested in modeling how users interact with each other in Reddit debates, to discover the most dominant opinions in a debate. To this end, we introduce a user-based model for analysis of Reddit debates. In this model, comments by users are grouped per user, describing their opinion in relation to the root comment of the debate, and users are represented with a single node in a weighted graph, where node's weights represent relevance of user's opinions and edges represent agreement or disagreement relationships between users throughout the debate. In this model, agreement or disagreement between the opinions of two users is defined by aggregating the set of single interactions that have occurred between them during the debate. In this work we present a skeptical aggregation model for this task. For measuring the relevance of user's opinions, we consider two models: one based on the score of all the user's comments and other based on the user's karma, as computed by the Reddit platform. We characterize the set of most dominant opinions with an argumentative-based model, using the information of disagreement between opinions and relevance of opinions.
\end{abstract}

Keywords. Reddit, user-based model, skeptical aggregation, argumentation-based reasoning, consensus analysis.

\section{Introduction}

Reddit (http://www.reddit.com/) is a social news aggregation, web content rating, and discussion website. Users submit content to the site such as links, text posts, and images, which are then voted up or down by other members who in turn, can comment on others' posts to continue the conversation. This online debating platform is widely used to create long and deep debates with comments and answers to comments, where, thanks to the almost unlimited text length of Reddit comments (40,000 characters), users can express their opinions more accurately compared to other online debating platforms such as Twitter that restricts the number of characters to 280 .

\footnotetext{
${ }^{1}$ Correspondence to: T. Alsinet. INSPIRES Research Centre, University of Lleida. C/Jaume II, 69. Lleida, Spain. Tel.: +34 973702734; E-mail: teresa.alsinet@udl.cat.
} 
The analysis of opinions on general and specialized social networks, has received a lot of attention on many application fields. For example, there is a vivid interest in the analysis of tourists' opinions [13,16,17], and similar efforts are being done on marketing $[5,9,7]$. In order to understand what are the major accepted and rejected comments in different domains by Reddit users, in a previous work [3], we have proposed a representation model for analysis of Reddit debates oriented to comments. The model considers that debates are two-sided, where some of the comments are in agreement with the root comment of the debate, and the rest of the comments are in disagreement. Then, debates are represented as bipartite graphs where edges indicate disagreement between comments of these two disjoint sets.

Our aim in this work is to progress in the analysis of the debates in Reddit that allow us to identify structural relations between users' opinions and to be able to discover the most dominant users' opinions in a debate, that is, the opinions that are most widely accepted among the different users. To be precise, we propose to model how different users interact with each other in Reddit debates and to perform an analysis of dominant users' opinions based on argumentation frameworks. To this end, we introduce a natural extension of our previous comment-oriented model [3], that we call user-based model for Reddit debates. In this new model, comments within a debate are grouped by users or authors, such that comments of the same users that describe their opinion along the debate are represented by single nodes in the graph, and edges stand for agreement or disagreement relationships between users' opinions in the debate. When we represent a debate grouping comments by users, interactions between different users can give rise to circular agreement and disagreement relationships, and the agreement or disagreement of a user in relation to the opinion of another user in the debate should be defined by aggregating the set of single interactions that have occurred between them during the debate. In this work, the aggregation follows a skeptical approach giving rise to neutral interactions when a user is simultaneously in agreement with one part of an other user's opinion an in disagreement with the rest, which is a key difference with the approach we defined in our past work on a user-based model for Twitter discussions [2] where support relationships between users were not considered.

The final goal of the new user-based model is to find a set of users' opinions that is consistent and is widely accepted by most of the users, following acceptance semantics that come from argumentation theory, in which consistency is defined as absence of disagreement from one accepted opinion to another one, when the first opinion is considered more relevant than the second one. So, we characterize the set of most dominant users' opinions as this set of widely accepted and consistent user's opinions, where we use ideal semantics [8] to define this set. To the best of our knowledge, this is the first work to use an argumentation approach to analyze the dominant users' opinions in Reddit debates. Previous work in other social networks, like Twitter, has considered also the study of interactions between users, mainly using the Twitter retweet graph [15,14], but these works do not consider the aggregation of user's comments. For Reddit, studies are centered towards analyzing different dynamic characteristics of the discussions [6], or like in our past work on characterizing user profiles [3], but always using a comment-oriented model.

Argumentation has also been explored as a cybersecurity tool [12] for helping making decisions where a balance has to be found between security, operability and cost. The main difference between this field and our framework is that arguments are not com- 
ments on a social network, but statements about system configuration, sensor data, etc. And, as in many others fields, there is some degree of conflicting information with different possible causes and solutions for a specific problem. It has been noted [11], however, that argumentation-based decisions can be manipulated by attacking key arguments to overturn the solution. In the same way, we are currently studying how to extend the user-based model to detect users that have deliberately manipulated a debate by means of their influence degree [3] and, possibly, other measures.

The rest of the paper is organized as follows. In Section 2, we recall [3] the commentbased model for Reddit debates. Then, in Section 3, we formalize the user-based model to analyze the global behavior of users in debates based on a skeptical sentiment analysis approach to assess the agreement and disagreement relationship between users. Finally, in Section 4, we define and test our argumentation-based reasoning system to compute the set of dominant user's opinions for a Reddit debate.

\section{Comment-based model for Reddit}

In this section, we present the computational structure we have already defined [3] to analyze the agreement between comments in Reddit debates. This approach considers that debates are two-sided, where some of the comments are in agreement with the root comment of the debate, and the rest of the comments are in disagreement. We reference this debate structure as comment-based model for a Reddit debate. Next, we first formalize the notions of comment and Reddit debate for a root comment.

A comment $c$ is a tuple $c=(m, u, s c)$, where $m$ is the text of the comment, $u$ is the user's identifier of the comment, and $s c \in \mathbb{Z}$ is the score of the comment.

Let $c_{1}=\left(m_{1}, u_{1}, s c_{1}\right)$ and $c_{2}=\left(m_{2}, u_{2}, s c_{2}\right)$ be two comments. We say that $c_{1}$ answers $c_{2}$ if $c_{1}$ is a reply to the comment $c_{2}$.

Let $r=\left(m_{r}, u_{r}, s c_{r}\right)$ be a comment such that $m_{r}$ contains a link to some news. A Reddit debate on the root comment $r$ is a non-empty set $\Gamma$ of Reddit comments such that $r \in \Gamma$ and every comment $c \in \Gamma, c \neq r, c$ answers a previous comment in $\Gamma$.

In Reddit, except for the root comment, each comment answers exactly one previous comment, usually by another user or author, and the score of a comment is computed as the sum of positive and negative votes that the comment has received. So, the score of a comment is negative whenever the comment has more negative votes than positive ones and positive, otherwise.

Given the structure of a Reddit debate $\Gamma$ on a root comment $r$, the next step is to extract the relationships between the comments in $\Gamma$. We represent $\Gamma$ as a labeled tree, where each comment gives rise to a node, edges denote answers between comments and are labeled with a value in the real interval $[-2,2]$. The label for an edge $\left(c_{1}, c_{2}\right)$ denotes the sentiment expressed in the text of the comment $c_{1}$ in response to the text of the comment $c_{2}$, so that, the value -2 denotes a total disagreement and the value 2 a total agreement. We use the sentiment value 0 to denote both answers expressing a neutral position with respect the opinion expressed in $c_{2}$, and answers expressing at the same time agreement with part of the opinion expressed in $c_{2}$, and disagreement with another part of $c_{2}$.

Formally, let $\Gamma$ be a Reddit debate on a root comment $r$. A Debate Tree (DebT) for $\Gamma$ is a tuple $\mathcal{T}=\langle C, r, E, L\rangle$ such that: (i) for every comment in $\Gamma$ there is a node in $C$, 
(ii) node $r \in C$ is the root node of $\mathcal{T}$, (iii) if $c_{1}$ answers $c_{2}$ then there is a directed edge $\left(c_{1}, c_{2}\right)$ in $E$, (iv) $L$ is a labeling function $L: E \rightarrow[-2,2]$, where the value assigned to an edge denotes the sentiment of the answer, from highly negative (-2) to highly positive (2), and (v) only the nodes and edges obtained by applying this process belong to $C$ and $E$, respectively.

Given a Reddit debate, we make its corresponding DebT using the Python Reddit API Wrapper (PRAW) ${ }^{1}$ to download its set of comments, and then we evaluate the sentiment for an edge $\left(c_{1}, c_{2}\right)$ in the DebT by means of the sentiment analysis software of [10] using the text of the comment $c_{1}$, where the value assigned denotes the sentiment of the answer, from highly negative (-2) to highly positive (2).

\section{User-based model for Reddit}

Our goal in this work is to introduce and investigate a suitable user-based model that allows us to represent how different users interact with each other in Reddit debates allowing us to discover the most dominant users' opinions. To this end, we group comments of a debate by user and we consider that the relationship between users' opinions of two users are defined from the agreement and disagreement relationships between the individual comments of these two users.

We consider debates in which every user's opinion is consistent (users do not contradict themselves) and where users are not self-referenced. That is, for each user $u$ and each pair of comments $c_{1}=\left(m_{1}, u, s c_{1}\right)$ and $c_{2}=\left(m_{2}, u, s c_{2}\right)$, we assume that messages $m_{1}$ and $m_{2}$ do not express neither conflicting nor inconsistent information, and that $c_{1}$ does not respond to $c_{2}$, nor $c_{2}$ to $c_{1}$.

Given a debate $\Gamma$ on a root comment $r$ with users' identifiers $\left\{u_{1}, \ldots, u_{n}\right\}$, we define the opinion of the user $u_{a} \in\left\{u_{1}, \ldots, u_{n}\right\}$, denoted $C_{a}$, as the set of comments of $u_{a}$ in $\Gamma$ except for the root comment $r$. If we consider the particular case of root users (the users who post the root comment), we notice that, when they do not participate through the debate, their opinion is empty, denoting that they have only posted the (root) news while staying passive throughout the debate. This is intentional, since the root comment plays a special role in the debate, setting its topic. Thus, in order to be considered a "true participant" on the debate, the root user should contribute during the discussion. Notice that the Reddit platform itself distinguishes between root and non-root comments, since it provides two different global user metrics, Post Karma and Comment Karma, where the first one corresponds to the points achieved by posting interesting news (root comments) and the second one corresponds to the points achieved from non-root comments (debate generated on some root comment).

Next we formalize the graph we propose to represent user-centered debates, called User Debate Graph, where the nodes are the users of the debate denoting their opinion with respect to the root comment and the edges denote interactions between users. In addition, we associate each edge of the graph with a weight representing the overall sentiment of the interactions between users.

Definition 1 (User Debate Graph) Let $\Gamma$ be a Reddit debate on a root comment $r$ with users' identifiers $\left\{u_{1}, \ldots, u_{n}\right\}$ and let $\mathcal{T}=\langle C, r, E, L\rangle$ be a DebT for $\Gamma$. A User Debate Graph (UDebG) for $\mathcal{T}$ is a tuple $\mathcal{G}=\langle\mathcal{C}, \mathcal{E}, \mathcal{L}\rangle$, where

\footnotetext{
${ }^{1}$ https://github.com/praw-dev/praw
} 
- $\mathcal{C}$ is the set of nodes of $\mathcal{G}$ defined as the set of users' opinions $\left\{C_{1}, \ldots, C_{n}\right\}$; i.e. $\mathcal{C}=\left\{C_{1}, \ldots, C_{n}\right\}$ with $C_{a}=\left\{\left(m, u_{a}, s c\right) \in \Gamma \mid\left(m, u_{a}, s c\right) \neq r\right\}$, for all users $u_{a} \in\left\{u_{1}, \ldots, u_{n}\right\}$.

- $\mathcal{E}$ is the set of edges of $\mathcal{G}$ defined as the set of interactions between different users in the debate; i.e. there is an edge $\left(C_{a}, C_{b}\right) \in \mathcal{E}$, with $u_{a}, u_{b} \in\left\{u_{1}, \ldots, u_{n}\right\}$ and $u_{a} \neq u_{b}$, iff there is $\left(c_{1}, c_{2}\right) \in E$ such that $c_{1} \in C_{a}$ and $c_{2} \in C_{b}$.

- $\mathcal{L}$ is a sentiment weighting scheme for $\mathcal{E}$; i.e. a map $\mathcal{L}: \mathcal{E} \rightarrow[-2,2]$ assigning to every edge $\left(C_{a}, C_{b}\right) \in \mathcal{E}$ a value in $[-2,2]$, that expresses the overall sentiment of the user $u_{a}$ regarding the comments of the user $u_{b}$ in the debate, from highly negative (-2) to highly positive (2). For each edge $\left(C_{a}, C_{b}\right) \in \mathcal{E}$, the value $\mathcal{L}\left(C_{a}, C_{b}\right)$ is meant to be computed from the individual sentiment of each answer in $C_{a}$ to a comment in $C_{b}$; i.e. from the set $\left\{L\left(c_{1}, c_{2}\right) \mid\left(c_{1}, c_{2}\right) \in E, c_{1} \in C_{a}\right.$ and $\left.c_{2} \in C_{b}\right\}$.

Only the nodes and edges obtained by applying this process belong to $\mathcal{C}$ and $\mathcal{E}$, respectively.

Notice that if a user only responds to the news of the debate (the root comment $r$ ), the user is mapped in the UDebG to a node in $\mathcal{C}$ with zero output degree denoting that the user starts no discussion with other users. In addition, users whose comments do not generate answers from other users are represented with nodes whose input degree is zero. Therefore, isolated nodes may appear in the UDebG that correspond to users who have neither generated nor participated in the debate, that is, users that have only answered to the news and whose opinions can be considered as accepted by all users since they have not been discussed yet.

In the UDebG, each node denotes a user's opinion and relationships between nodes are mined from the prevailing sentiment among the aggregated comments of those nodes. Moreover, a user can answer comments of different users in a debate, and thus, can agree or disagree with several users. However, if a user $u_{a}$ answers several comments of a same user $u_{b}$, the set of interactions between them is represented with a single edge $\left(C_{a}, C_{b}\right)$ in $\mathcal{E}$ and with a single sentiment value $\mathcal{L}\left(C_{a}, C_{b}\right)$, which is meant to denote the overall sentiment of agreement or disagreement of the user $u_{a}$ with respect to the user $u_{b}$.

Analogously, as we did in the previous section to define the sentiment relation between comments, we propose here to use a skeptical sentiment scheme for weighting the agreement or disagreement relation between users. Our skeptical approach is based on stating that a user agrees or disagrees with another user if and only if one can be completely sure of it.

Our aim is to define a sentiment weighting scheme $\mathcal{L}: \mathcal{E} \rightarrow[-2,2]$ for edges in $\mathcal{E}$, by combining, in a skeptical way, the individual sentiment values assigned to the responses between comments; i.e. for any pair of users $u_{a}$ and $u_{b}$ with opinions $C_{a}$ and $C_{b}$, respectively, and with $\left(C_{a}, C_{b}\right) \in \mathcal{E}, \mathcal{L}\left(C_{a}, C_{b}\right)$ is defined from the values in $\left\{L\left(c_{1}, c_{2}\right) \mid c_{1} \in C_{a}\right.$ and $\left.c_{2} \in C_{b}\right\}$. To be precise, we define the skeptical sentiment relation of the opinion $C_{a}$ of the user $u_{a}$ with respect to the opinion $C_{b}$ of the user $u_{b}$ as follows:

- $u_{a}$ agrees with $u_{b}$, denoted as $C_{a} \rightarrow C_{b}$, iff all answers from the user $u_{a}$ to the user $u_{b}$ are positive, i.e. $C_{a} \rightarrow C_{b}$ iff $L\left(c_{1}, c_{2}\right)>0$, for all $\left(c_{1}, c_{2}\right) \in E$ with $c_{1} \in C_{a}$ and $c_{2} \in C_{b}$; 
- $u_{a}$ disagrees with $u_{b}$, denoted as $C_{a} \rightsquigarrow C_{b}$, iff all answers from the user $u_{a}$ to the user $u_{b}$ are negative, i.e. $C_{a} \rightsquigarrow C_{b}$ iff $L\left(c_{1}, c_{2}\right)<0$, for all $\left(c_{1}, c_{2}\right) \in E$ with $c_{1} \in C_{a}$ and $c_{2} \in C_{b}$; and

- $u_{a}$ is neutral with respect to $u_{b}$, otherwise.

The neutral relation between users denotes both interactions expressing an overall neutral position of the user $u_{a}$ with respect to the comments of the user $u_{b}$, and interactions expressing at the same time agreement with some of the comments in $C_{b}$, and disagreement with the rest. Moreover, as we are assuming a skeptical scheme, for both agreement and disagreement relationships between users we aggregate the overall sentiment as the minimum value of the individual answers which corresponds to a pessimistic interpretation of the degree of agreement and disagreement among users. This aggregation model tries to represent the fact that in a debate with multiple negative responses from one user to another, a pessimistic analysis will focus on the most negative response, and in the case of multiple positive responses, on the softest positive response.

Formally, we define the skeptical sentiment weighting scheme $\mathcal{L}: \mathcal{E} \rightarrow[-2,2]$ for edges $\left(C_{a}, C_{b}\right) \in \mathcal{E}$ based on a pessimistic aggregation model of the degree of agreement or disagreement among users' comments as follows:

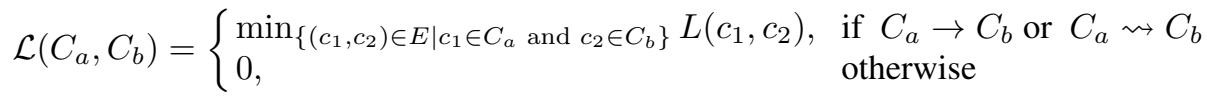

Notice that for agreement relationships $\left(C_{a} \rightarrow C_{b}\right)$ we consider the least agreement value (i.e. the value closest to 0$)$, while for disagreement relationships $\left(C_{a} \rightsquigarrow C_{b}\right)$ we consider the highest disagreement value (i.e. the value closest to -2 ). We use the neutral sentiment value 0 for neutral relations.

The UDebG for a given DebT may contain cycles. These cycles provide fundamental information about the different relationships that are established from the interactions between different users. In this sense, aggregating the information by users allows us to identify the set of users whose opinions are accepted by most users, the authors involved in a circular argumentative discussion, or the most controversial users.

\section{Discovering dominant users' opinions}

Once we have introduced the user-based model of debates in Reddit, the next step is to characterize the set of the most dominant user's opinions among the users' opinions of the debate. To this end, we extend the argumentation-based reasoning system we have already developed [1] to analyze discussions in Twitter, to deal here with Reddit debates to find a set of users' opinions that is consistent and is widely accepted by most of the users, following acceptance semantics that come from argumentation theory, in which consistency is defined as absence of disagreement from one accepted opinion to another one, when the first opinion is considered more relevant than the second one. The approach, described in the rest of the section, consists of mapping a UDebG graph for a Reddit debate to a value-based abstract argumentation framework and to use ideal semantics to define the most dominant users' opinions. 


\subsection{The argumentation-based reasoning system}

Given a UDebG with a sentiment scheme $\mathcal{L}$ for weighting the agreement or disagreement relationship between users, we build a corresponding value-based argumentation framework where arguments represent users' opinion and attacks between arguments represent disagreement relationships between users. Value-based abstract argumentation, introduced by Bench-Capon [4], is an extension of abstract argumentation with a valuation function $\mathrm{Val}$ for arguments taking values on a set $R$ equipped with a (possibly partial) preference relation Valpref.

Definition 2 (VAF for a UDebG) Let $\Gamma$ be a Reddit debate on a root comment $r$ with users identifiers $\left\{u_{1}, \ldots, u_{n}\right\}$ and let $\mathcal{G}=\langle\mathcal{C}, \mathcal{E}, \mathcal{L}\rangle$ be a UDebG for $\Gamma$. A Value-based Argumentation Framework $(V A F)$ for $\mathcal{G}$ is a tuple $\mathcal{F}=\langle\mathcal{C}$, attacks, $R$, Val, Valpref $\rangle$, where

- each node or user's opinion in $\mathcal{C}=\left\{C_{1}, \ldots, C_{n}\right\}$ results in an argument in the $V A F \mathcal{F}$,

- attacks is an irreflexive and asymmetric binary relation on $\mathcal{C}$ which corresponds to the set of disagreement edges between users' opinions in $\mathcal{E}$ :

$$
\text { attacks }=\left\{\left(C_{a}, C_{b}\right) \in \mathcal{E} \mid \mathcal{L}\left(C_{a}, C_{b}\right)<0\right\}
$$

i.e. the user $u_{a}$ attacks the user $u_{b}$ if and only if $C_{a} \rightsquigarrow C_{b}$,

- $R$ is a non-empty set of values that models the social support of users,

- Val $: \mathcal{C} \rightarrow R$ is a valuation function that assigns social support values in $R$ to arguments or users' opinion in $\mathcal{C}$, and

- Valpref $\subseteq R \times R$ is an order relation (transitive, irreflexive and asymmetric) on $R$ reflecting preferences between social support values in $R$.

Once we have the VAF $\mathcal{F}$ associated with a UDebG $\mathcal{G}$, we consider ideal semantics, formalized by Dung et al. [8], to define the set of widely accepted users' opinions from the debate, also called the solution for the debate. Ideal semantics guarantees that users' opinions in the solution represent the maximal set of acceptable user's opinions, in the sense that contrary opinions are weaker or they are defeated by other accepted stronger user's opinions, and are consistent, in the sense that there are no defeats between user opinion's in the solution, as we formally describe next.

In our approach, a defeat, or effective attack between two users' opinions, is defined relative to an attack strength threshold $\alpha \in[0,2]$, which characterizes the minimum degree of disagreement of one user's opinion regarding another user's opinion, and relative to the strength (social support) of the user's opinions.

Formally, let $\mathcal{F}=\langle\mathcal{C}$, attacks, $R$, Val, Valpref $\rangle$ be a VAF for a UDebG $\mathcal{G}=\langle\mathcal{C}, \mathcal{E}, \mathcal{L}\rangle$ and let $\alpha \in[0,2]$ be an attack strength threshold. The defeats relation over users based on the Val function, the Valpref relation and the threshold $\alpha$ is defined as follows:

$$
\text { defeats }=\left\{\left(C_{a}, C_{b}\right) \in \text { attacks } \mid\left(\operatorname{Val}\left(C_{b}\right), \operatorname{Val}\left(C_{a}\right)\right) \notin \text { Valpref and }\left|\mathcal{L}\left(C_{a}, C_{b}\right)\right|>\alpha\right\} ;
$$

i.e. the user $u_{a}$ defeats the user $u_{b}$ if and only if (i) $C_{a} \rightsquigarrow C_{b}$, (ii) the social support value of $C_{b}$ is not preferred over the social support value of $C_{a}$ and (iii) the highest degree of disagreement of comments in $C_{a}$ with respect to comments in $C_{b}$ is greater than $\alpha$. 
Notice that the attack strength threshold $\alpha=0$ has no pruning effect on the defeats relation.

Based on the defeats relation, we say that a set of users' opinion $S \subseteq \mathcal{C}$ is conflictfree if for all $C_{a}, C_{b} \in S,\left(C_{a}, C_{b}\right) \notin$ defeats. Moreover, a conflict-free set of users' opinion $S \subseteq \mathcal{C}$ is maximally admissible if for all $C_{a} \notin S, S \cup\left\{C_{a}\right\}$ is not conflictfree and, for all $C_{b} \in S$, if $\left(C_{a}, C_{b}\right) \in$ defeats, then there exists $C_{d} \in S$ such that $\left(C_{d}, C_{a}\right) \in$ defeats. Finally, the solution for a debate is the largest admissible conflictfree set of users' opinion $S \subseteq \mathcal{C}$ in the intersection of all maximally admissible conflictfree sets.

We select this semantics to define the solution for a debate, because it represents a maximally admissible set of conflict-free users' opinions, such that they defend against attacks outside this set, and they are included in any admissible set of users' opinions. This set therefore represents a kind of maximum set of widely accepted user's opinions between all the possible admissible sets of users' opinions. Dung et al. [8] prove that this solution is unique.

\subsection{Implementation and analysis of results}

As for the implementation purposes of our argumentation-based reasoning system, we instantiate the elements of a VAF $\mathcal{F}=\langle\mathcal{C}$, attacks, $R$, Val, Valpref $\rangle$ as follows: (i) the set $R$ of social support values of users is instantiated to the set of natural numbers $\mathbb{N}$, (ii) the preference relation Valpref on $R$ is instantiated to the natural order on $\mathbb{N}$, and (iii) the valuation function Val maps a user's opinion $C_{a} \in \mathcal{C}$ to a natural number in $\mathbb{N}$. With the aim of stratifying users with really significant levels of relevance in the debate platform, the Val function stratifies the social relevance of users' opinions by assigning zero to a user's opinion with zero or negative support and, for positive support, considers that one user's opinion has more social relevance than another only if the support is at least ten times bigger. So, we consider the following definition for the valuation function Val: $\operatorname{Val}\left(C_{a}\right)=\left\lfloor\log _{10} \operatorname{support}\left(\mathrm{C}_{\mathrm{a}}\right)\right\rfloor+1$, if $\operatorname{support}\left(\mathrm{C}_{\mathrm{a}}\right) \geq 1$ and $\operatorname{Val}\left(C_{a}\right)=0$, otherwise, where support $\left(\mathrm{C}_{\mathrm{a}}\right)$ is some social support metric for users' opinions available on the Reddit platform.

To compute the social support metric for users' opinions, we consider two different user parameters available on the Reddit platform. On the one hand, we consider a metric based on the Comment Karma global user parameter, refereed as karma $\left(C_{a}\right)$ and computed as the Comment Karma of the user $u_{a}$ available in Reddit. In this case, active users that post interesting comments are considered more relevant than users who are not very active or have little impact on the platform. On the other hand, we also consider a social support metric based on the sum of the scores of all the comments of a user in the debate under analysis and computed as score $\left(C_{a}\right)=\sum_{\left\{\left(m, u_{a}, s c\right) \in C_{a}\right\}} s c$. In this case, users whose comments are valued or rated positively are considered more relevant than users with comments with negative scores.

Although the computational complexity of computing ideal semantics for general VAFs is even higher than NP, there are some cases that can be solved within polynomial time. With the goal of being able to solve instances with a scalable approach, we have developed a distributed algorithm [1] that computes within linear time the solution of a VAF for two special cases: graphs with no even cycles and bipartite graphs.

The implementation of the distributed algorithm consists of a pre-processing step and the actual distributed computation of the solution of the VAF resulting of the pre- 
processing. The pre-processing step prunes all attacks that are not effective based on the weighting scheme of edges and the valuation function of nodes. The distributed computation algorithm is based on the computation model of Pregel. This model is appropriate for our problem, because the input for a Pregel algorithm is a directed graph, where the nodes can be in different states, and the goal of a distributed algorithm in Pregel is to compute the state of each node based on the state of the nodes' neighbors.

Also, we have already shown [1] that for a VAF with a general graph, the output provided by the distributed algorithm coincides with the so-called grounded extension or solutionwhich, in turn, is always a subset of the ideal semantics solution (the solution we want to find). So, we can safely use the distributed algorithm as a sound approximation algorithm for such cases that are in principle not solvable by the algorithm (i.e. graphs with even cycles that are not bipartite).

Figure 1 shows the solutions for a Reddit debate of the subreddit World News (r/worldnews $)^{2}$ based on the score (left) and karma metrics (right). Each user's opinion is represented as a node and each relationship between users' opinions is represented as an edge. The edges are colored in black, green and red to denote neutral, agreement and disagreement relationships between users' opinions, respectively. The nodes colored in blue are the users' opinions in the solution and the nodes colored in gray are the rejected ones, where the darkness of the color is directly proportional to the value of the score and karma metrics of each user with respect to the maximum value.

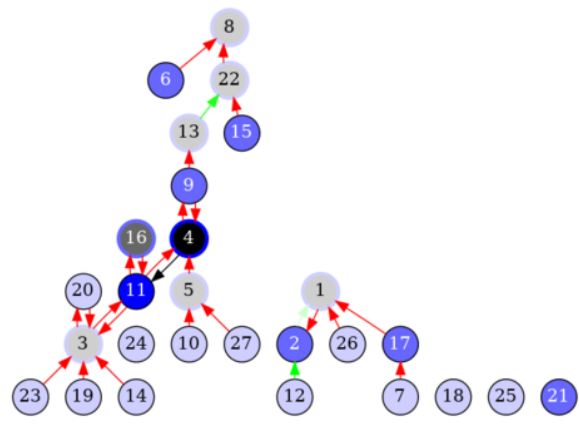

(a) Solution based on the score metric.

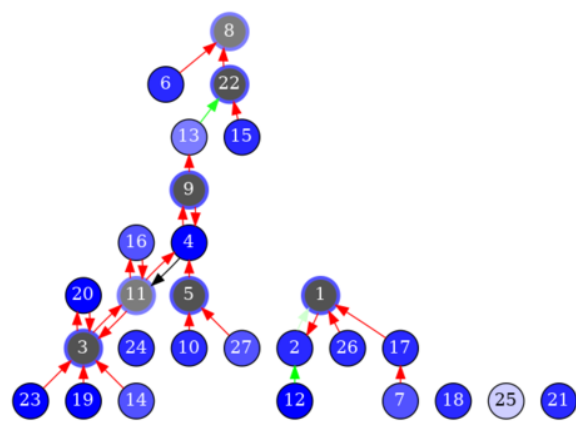

(b) Solution based on the karma metric.

Figure 1. Solutions for a Reddit debate with attack strength threshold $\alpha=0.5$.

For the score metric, the solution contains 18 of the 27 users' opinions. We observe that from the users that concentrate more interactions $(3,4$, and 11$)$, the solution includes only user 11 , because user 11 has a score bigger than the ones of the users 3 and 16, so although there are mutual attacks between 11 and 3 and 11 and 16, only the ones from user 11 are defeats. For the karma metric, the solution changes because the relevance of some users changes. This time, we have that user 16 , that attacks user 11 , is more relevant, so user 16 defeats user 11, that in turn causes that user 4 is accepted in the solution. This impacts also on other users. For example, user 9 is not accepted because the accepted user 4 defeats user 9, and this in turn produces the acceptance of user 13. So, changes in the way of measuring the relevance of user's opinions may have a relevant contribution on the set of accepted opinions.

\footnotetext{
${ }^{2}$ https://www.reddit.com/r/worldnews/comments/f 62 i 35
} 
As future work, we plan to study the suitability of other schemes for the aggregation of individual interactions between two users, when computing the sentiment weighting scheme, as well as different approaches for the definition of the users' relevance.

Acknowledgments This work was partially funded by Spanish Project PID2019-111544GBC22, by the European Union's Horizon 2020 Research and Innovation Programme under Grant Agreement 723596 and Grant Agreement 768824, and by 2017 SGR 1537.

\section{References}

[1] Alsinet, T., Argelich, J., Béjar, R., Cemeli, J.: A distributed argumentation algorithm for mining consistent opinions in weighted twitter discussions. Soft Comput. 23(7), 2147-2166 (2019)

[2] Alsinet, T., Argelich, J., Béjar, R., Esteva, F., Godo, L.: A probabilistic author-centered model for twitter discussions. In: Proceedings of IPMU Conference. vol. 854, pp. 683-695. Springer (2018)

[3] Alsinet, T., Argelich, J., Béjar, R., Martínez, S.: Measuring user relevance in online debates through an argumentative model. Pattern Recognition Letters 133, 41-47 (2020)

[4] Bench-Capon, T.J.M.: Value-based argumentation frameworks. In: Proceedings of NMR International Workshop. pp. 443-454 (2002)

[5] Burton, S., Soboleva, A.: Interactive or reactive? marketing with Twitter. Journal of Consumer Marketing 28(7), 491-499 (2011)

[6] Choi, D., Han, J., Chung, T., Ahn, Y.Y., Chun, B.G., Kwon, T.T.: Characterizing conversation patterns in reddit: From the perspectives of content properties and user participation behaviors. In: Proceedings of COSN Conference. pp. 233-243. ACM (2015)

[7] Chu, S.C., Kim, Y.: Determinants of consumer engagement in electronic word-of-mouth (ewom) in social networking sites. International Journal of Advertising 30(1), 47-75 (2011)

[8] Dung, P.M., Mancarella, P., Toni, F.: Computing ideal sceptical argumentation. Artif. Intell. 171(10-15), 642-674 (2007)

[9] Jansen, B.J., Zhang, M., Sobel, K., Chowdury, A.: Twitter power: Tweets as electronic word of mouth. Journal of the American Society for Information Science and Technology 60(11), 2169-2188 (2009)

[10] Manning,C.D.,Surdeanu,M.,Bauer,J.,Finkel,J.,Bethard,S.J.,McClosky,D.:TheStanford CoreNLP natural language processing toolkit. In: Association for Computational Linguistics (ACL) System Demonstrations. pp. 55-60 (2014)

[11] Martinelli, F., Santini, F.: Debating cybersecurity or securing a debate? In: International Symposium on Foundations and Practice of Security. pp. 239-246. Springer (2014)

[12] Martinelli, F., Santini, F., Yautsiukhin, A.: Network security supported by arguments. In: 2015 13th Annual Conference on Privacy, Security and Trust (PST). pp. 165-172. IEEE (2015)

[13] McCarthy, L., Stock, D.: How travelers use online and social media channels to make hotelchoice decisions. Cornell Hospitality Reports 10(18), pp. 6-18 (2010)

[14] Motamedi, R., Rezayi, S., Rejaie, R., Willinger, W.: On characterizing the twitter elite network. In: Proceedings of ASONAM Conference. pp. 234-241. IEEE (2018)

[15] ten Thij, M., Ouboter, T., Worm, D., Litvak, N., van den Berg, H., Bhulai, S.: Modelling of trends in twitter using retweet graph dynamics. In: Algorithms and Models for the Web Graph. pp. 132-147. Springer (2014)

[16] Villatoro, D., Serna, J., Rodríguez, V., Torrent-Moreno, M.: The tweetbeat of the city: Microblogging used for discovering behavioural patterns during the mwc2012. In: Citizen in Sensor Networks, LNCS, vol. 7685, pp. 43-56. Springer (2013)

[17] Williams, N.L., Inversini, A., Buhalis, D., Ferdinand, N.: Community crosstalk: an exploratory analysis of destination and festival ewom on Twitter. Journal of Marketing Management 31(9-10), 1113-1140 (2015) 